\title{
Reclassements des plants de hêtre (Fagus sylvatica $L$ ) au cours de leur développement dans une régénération naturelle, équienne, âgée de 18 ans
}

\author{
B Thiébaut 1, 2*, B Comps 3, M Rucart 1, \\ S Soroste 1, C Ntsame Okwo 1 \\ 1 Université des sciences et techniques du Languedoc, Institut de botanique, \\ 163, rue Auguste Broussonnet, 34000 Montpellier; \\ 2 CNRS, Centre Louis Emberger, BP 5051, 34033 Montpellier cedex; \\ ${ }^{3}$ Université de Bordeaux I, département de biologie des végétaux ligneux, \\ avenue des Facultés, 33405 Talence, France
}

(Reçu le 15 juillet 1991; accepté le 19 novembre 1991)

\begin{abstract}
Résumé - Dans une régénération naturelle de hêtre, équienne, âgée de 18 ans et située sous 3 éclairements, la structure est pluristrate à la lumière et en demi-lumière et monostrate à l'ombre. Le développement des plants en hauteur a été reconstitué chaque année, le long de leur tige principale, grâce aux cicatrices laissées sur l'écorce par le bourgeon apical chaque année.

La présence ou l'absence d'une stratification ne semblent pas avoir d'influence sur les reclassements et les recrutements au cours des 18 ans, ni sur la stabilité du dernier classement.
\end{abstract}

régénération naturelle / Fagus sylvatica $=$ hêtre / stratification / croissance en hauteur / reclassement

Summary - Annual classification of beech seedlings (Fagus sylvatica $L$ ) in an even-aged, 18-year-old and natural beech regeneration. In an even-aged 18-year-old natural beech regeneration, the height growth of young plants has been analysed on the main stem under 3 daylight intensities: full-light, half-light and shade. The structure showed 3, 2 and 1 layer(s) respectively under these light conditions. The annual classification of seedlings according to their total height over an 18-year period was found to be influenced by light conditions and vigour at 18 years old, but not by the structure of the regeneration.

natural seedling / Fagus sylvatica = beech / stratification / height growth / classification

* Correspondance et tirés à part 


\section{INTRODUCTION}

Dans les régénérations naturelles du hêtre, une structure verticale se manifeste physionomiquement par l'apparition d'une strate (= structure monostrate) ou de plusieurs strates (= structure pluristrate) qui correspondent à des niveaux de forte densité du feuillage. Au massif de l'Aigoual (Cévennes, Sud-Est de la France: $44^{\circ} 20^{\prime} \mathrm{N}, 3^{\circ} 60^{\prime} \mathrm{E}$ ), dans la forêt du Lingas, nous avons observé une structure pluristrate à la lumière et en demi-lumière et une structure monostrate à l'ombre, dans une régénération équienne de 18 ans (Thiébaut et al, 1992).

Ces stratifications sont souvent comparées à des hiérarchies sociales où la strate supérieure représenterait le peuplement principal et les autres strates des peuplements subordonnés (Falcone et al, 1986; Lanier, 1986; Schütz, 1990). Ce point de vue se justifie si la stratification a une influence sur le développement des plants. Dans la régénération étudiée nous avons constaté (Thiébaut et al, 1992):

- que la stratification agit sur la vigueur, la forme et le mode de croissance des individus à la lumière et en demi-lumière;

- que les différences de développement sont plus importantes entre les strates (variation interstrate) qu'à l'intérieur de celles-ci variation intrastrate) uniquement à la lumière;

- et que les strates se distinguent précocement et se maintiennent, même si la croissance annuelle diminue avec l'âge à cause de la densité élevée.

Cependant, nous n'avions pas encore examiné l'influence de la stratification sur les reclassements des plants, chaque année. C'est la question abordée ici.

\section{MÉTHODES}

La forêt du Lingas est située à $1100 \mathrm{~m}$ d'altitude sur un terrain granitique. Des parcelles ont été mises en régénération après une coupe d'éclaircie en 1968 et, jusqu'en 1986 au moment des observations, aucune opération culturale n'a été effectuée dans le peuplement.

\section{Description du dispositif}

La régénération naturelle a une répartition irrégulière. Dans la zone étudiée, elle est équienne et âgée de 18 ans. La densité peut atteindre 300 individus $/ \mathrm{m}_{2}$ (Thiébaut et al, 1992).

Un transect continu a été installé sous 3 éclairements à l'ombre, en demi-lumlère et en pleine lumière. Aux 2 extrémités du transect, de la lumière vers l'ombre, l'extinction de l'éclairement atteint $90 \%$ vers midi, par temps clair, en phénophase feuillée, entre 400 et $700 \mathrm{~nm}$. Les autres conditions du milieu : sol, microrelief et végétation adventice paraissent homogènes. En principe, tous les individus touchant un ruban métallique tendu sur $55 \mathrm{~m}$ ont été retenus pour étudier leur développement au cours de ces 18 années.

Cependant, seuls les plants sans dommages apparents ont été analysés : 390 sur 403 . Et les individus qui ont disparu avant 1986 n'ont évidemment pas été pris en compte. Mais les densités élevées observées ici laissent supposer que leur nombre a été certainement faible parmi les grands plants, compétitifs, alors qu'il a pu être plus élevé parmi les plus petits, peu compétitifs. Donc le biais dû à la disposition de certains individus doit être faible sur le développement morphologique des plants étudiés.

Les reclassements des plants se produisent d'une année à l'autre, selon leur hauteur relative dans un collectif d'individus. Pour examiner les effets respectifs de la stratification et de l'éclairement, nous avons évité les parties du transect soumises à des luminosités intermédiaires entre 2 éclairements différents. Ici, nos observations ont donc été limitées à 3 portions du transect, situées respectivement au centre des zones exposées à la lumière, en demi-lumière et à l'ombre. Le nombre total d'individus étudiés est de 290 , dont 100 à la lumière répartis entre 3 strates (40 dans la strate supérieure $L_{1}, 30$ dans la strate intermédiaire $L_{2}, 30$ dans la strate inférieure $L_{3}$ ), 130 en demi-lumière partagés entre 2 strates (70 dans la strate supérieure $l_{1}$ ), 60 dans la strate inférieure $\mathrm{I}_{2}$ ) et 60 à l'ombre ( 1 strate $\mathrm{O}_{1}$ ). 


\section{Analyse morphologique des individus}

Le développement en hauteur des jeunes hêtres a été décrit le long de leur tige principale. Les cicatrices laissées sur l'écorce par le bourgeon apical permettent de repérer exactement la base de toutes les pousses annuelles, la première pousse étant indiquée par les cicatrices opposées-décussées des 2 cotylédons et des 2 premiers bourgeons axillaires. La croissance de la tige principale a pu ainsi être reconstituée chaque année pendant 18 ans, à l'aide de la longueur de la pousse annuelle et de la hauteur totale, estimée en cumulant les longueurs des pousses précédentes.

\section{Reclassements des plants selon leur hauteur totale chaque année}

L'identité des plants et leur croissance étant connues, chaque année ils ont été rangés dans des classes de hauteur ayant un effectif égal à 10 individus. Nous avons suivi leurs déplacements d'une classe à l'autre entre 2 années consécutives et noté le moment où leur classement devient définitif dans leur strate-86 ou dans leur classe-86 (= recrutement). L'importance de ces mouvements peut être appréciée par le nombre de classes impliquées dans ces changements.

Les reclassements des plants peuvent être décrits selon plusieurs points de vue, en fonction de leur vigueur à 18 ans, c'est-à-dire selon leur strate-86 et selon leur classe-86, ou bien en fonction des individus ou des années. Sans développer ici toutes les analyses possibles, nous allons essayer de caractériser le dynamisme de la régénération au niveau des classements annuels, des recrutements puis des mouvements.

\section{RÉSULTATS}

\section{Classements annuels selon la strate des plants en 1986}

\section{À la lumière}

La première année, 21 plants sur 40 $(52,5 \%)$ appartenant à $\left(L_{1}\right)$ se trouvent dans les 4 plus grandes classes correspondant à cette strate, tableau I; 10 plants sur $30(33,3 \%)$ de $\left(L_{2}\right)$ sont dans les 3 classes moyennes, tableau $\mathrm{II}$; et seulement 6 plants sur $30(20,0 \%)$ de $\left(L_{3}\right)$ sont dans les 3 classes inférieures, tableau III. Puis ces proportions augmentent au cours des années pour atteindre $100 \%$, en 1982 pour les plants de $\left(L_{1}\right)$ et en 1986 pour les plants de $\left(L_{2}\right)$ et de $\left(L_{3}\right)$. Dans les 3 strates86 , la proportion des plants inclus dans leurs classes respectives augmente brutalement entre 1977 et 1978. Ces années jouent un rôle charnière important à cause de l'ampleur et du caractère définitif des reclassements qui se produisent entre elles. Les répartitions annuelles observées s'écartent significativement d'une distribution régulière des plants après 1977 .

Dans les 3 strates-86, les classements annuels évoluent conformément aux 3 phases de développement décrites précédemment (Thiébaut et al, 1992). De 1969 à 1973, pendant la phase d'installation, les plants se rencontrent à peu près dans toutes les classes de hauteur quelle que soit leur strate définitive en 1986. Entre 1973 et 1978, pendant la phase de différenciation, leur répartition se resserre de manière significative sur leurs classes en 1986. Enfin, pendant la phase de stabilisation, ils saturent définitivement leurs classes définitives respectives.

\section{En demi-lumière}

Sous cet éclairement, les classements des plants suivent la même évolution qu'à la lumière. Leur recrutement dans une strate86 s'ouvre largement à toutes les classes au début de leur développement, puis se resserre progressivement sur les classes correspondant à leur strate en 1986. Mais ici, le regroupement des individus est moins rapide et l'on observe aucun changement particulier entre 1977 et 1978. 
Tableau I. Lumière, classements annuels des 40 plants de la strate supérieure selon 10 classes de hauteurs. I: phase d'installation, II: de différenciation, III: de stabilisation (Thiébaut et al, 1992). Proportion des 40 plants de la strate supérieure observés dans les 4 premières classes, correspondant à cette strate (\%). $\chi^{2}$ : comparaison entre la répartition observée et une répartition régulière des 40 plants, en opposant les effectifs dans les 4 premières classes à celui des 6 dernières.

\begin{tabular}{|c|c|c|c|c|c|c|c|c|c|c|c|c|c|c|}
\hline \multirow[t]{2}{*}{ Années } & \multirow[t]{2}{*}{ Ages } & \multirow[b]{2}{*}{1} & \multirow[b]{2}{*}{2} & \multicolumn{6}{|c|}{ Classes de tailles décroissantes } & \multirow[b]{2}{*}{9} & \multirow{2}{*}{\multicolumn{2}{|c|}{$10^{(\%)}$}} & & \multirow[t]{2}{*}{$\left(\chi^{2}\right)$} \\
\hline & & & & 3 & 4 & 5 & 6 & 7 & 8 & & & & & \\
\hline \multicolumn{15}{|l|}{ III } \\
\hline 1986 & 18 & 10 & 10 & 10 & 10 & - & - & - & - & - & - & 100,0 & 100,0 & **** \\
\hline 1985 & 17 & 10 & 10 & 10 & 10 & - & - & - & - & - & - & 100,0 & 96,6 & *** \\
\hline 1984 & 16 & 10 & 10 & 10 & 10 & - & - & - & - & - & - & 100,0 & 93,3 & *** \\
\hline 1983 & 15 & 10 & 10 & 10 & 10 & - & - & - & - & - & - & 100,0 & 93,3 & *** \\
\hline \multicolumn{15}{|l|}{ II } \\
\hline 1982 & 14 & 10 & 10 & 10 & 10 & - & - & - & - & - & - & 100,0 & 93,3 & $\star \star \star \star$ \\
\hline 1981 & 13 & 10 & 10 & 10 & 7 & 3 & - & - & - & - & - & 92,5 & 86,6 & $\star * \star \star$ \\
\hline 1980 & 12 & 10 & 10 & 10 & 6 & 4 & - & - & - & - & - & 90,0 & 83,3 & **** \\
\hline 1979 & 11 & 10 & 10 & 9 & 7 & 4 & - & - & - & - & - & 90,0 & 83,3 & 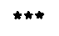 \\
\hline 1978 & 10 & 10 & 10 & 8 & 8 & 4 & - & - & - & - & - & 90,0 & 83,3 & **** \\
\hline 1977 & 9 & 8 & 6 & 8 & 7 & 4 & 4 & 2 & 1 & - & - & 72,5 & 33,3 & ** \\
\hline 1976 & 8 & 8 & 10 & 7 & 6 & 3 & 2 & 3 & 1 & - & - & 77,5 & 40,0 & ** \\
\hline 1975 & 7 & 6 & 9 & 7 & 6 & 3 & 2 & 2 & 4 & 1 & - & 70,0 & 36,6 & ** \\
\hline 1974 & 6 & 10 & 6 & 8 & 3 & 3 & 3 & 4 & 1 & 1 & 1 & 67,5 & 30,0 & NS \\
\hline \multicolumn{15}{|l|}{1} \\
\hline 1973 & 5 & 10 & 7 & 5 & 4 & 3 & 3 & 5 & 2 & 1 & - & 65,0 & 26,6 & $*$ \\
\hline 1972 & 4 & 10 & 6 & 4 & 3 & 6 & 2 & 3 & 5 & 1 & - & 57,5 & 26,6 & NS \\
\hline 1971 & 3 & 7 & 5 & 7 & 4 & 3 & 4 & 2 & 3 & 3 & 2 & 57,5 & 30,0 & NS \\
\hline 1970 & 2 & 7 & 7 & 3 & 5 & 4 & 3 & 3 & 3 & 4 & 1 & 55,0 & 36,6 & NS \\
\hline 1969 & 1 & 8 & 5 & 3 & 5 & 3 & 4 & 1 & 4 & 3 & 4 & 52,5 & 20,0 & NS \\
\hline
\end{tabular}

\section{Classements annuels selon l'éclairement}

Sous chaque éclairement, les corrélations linéaires ont été calculées sur les classements annuels entre toutes les années, prises 2 à 2 (fig 1). Un coefficient faible traduit des reclassements importants entre 2 années successives et un coefficient élevé une stabilité des plants.

Les reclassements se produisent essentiellement au début du développement. Au cours d'une première période, relativement longue et bien marquée à la lumière mais brève et peu nette de demi-lumière et à l'ombre, de fortes corrélations entre plusieurs années consécutives traduisent un classement provisoire des plants. À la lumière, celui-ci est remis en cause entre 1977-1978, puis après 1978 les plants paraissent définitivement classés jusqu'en 1986 (coefficients > 0,900). En demilumière la rupture se produit plus tôt entre 1973-1974 et par la suite, les plants ne paraissent définitivement classés qu'après 1981. Enfin, à l'ombre la rupture s'établit entre 1974-1975 et les plants ne sont classés qu'après 1983. Ainsi le classement définitif se met en place tôt et se produit rapi- 
Tableau II. Lumière, classements annuels des 30 plants de la strate intermédiaire selon 10 classes de hauteurs. I: phase d'installation, II: de différenciation, III: de stabilisation (Thiébaut et al, $1992 \mathrm{~b}$ ). Proportion des 30 plants de la strate intermédiaire observés dans les 3 classes moyennes, correspondant à cette strate (\%). $\chi^{2}$ : comparaison entre la répartition observée et une répartition régulière des 30 plants, en opposant les effectifs dans les 3 classes moyennes $(5+6+7)$ à celui des 7 autres classes $(1+2+3+4+8+9+10)$.

\begin{tabular}{llllllllllll}
\hline Années Ages & \multicolumn{1}{c}{ Classes de tailles décroissantes } & & & $(\%)$ & $\left(\chi^{2}\right)$
\end{tabular}

\begin{tabular}{|c|c|c|c|c|c|c|c|c|c|c|c|c|c|c|}
\hline II & & & & & & & & & & & & & & \\
\hline 1986 & 18 & - & - & - & - & 10 & 10 & 10 & - & - & - & 100,0 & 100,0 & "*** \\
\hline 1985 & 17 & - & - & - & - & 10 & 10 & 9 & 1 & - & - & 96,6 & 96,6 & $* * * *$ \\
\hline 1984 & 16 & - & - & - & - & 10 & 10 & 8 & 2 & - & - & 93,3 & 93,3 & $* * *$ \\
\hline 1983 & 15 & - & - & - & - & 10 & 10 & 8 & 2 & - & - & 93,3 & 93,3 & $* * *$ \\
\hline II & & & & & & & & & & & & & & \\
\hline 1982 & 14 & - & - & - & - & 10 & 10 & 8 & 2 & - & - & 93,3 & 93,3 & \\
\hline 1981 & 13 & - & - & - & 7 & 3 & 9 & 7 & 3 & 1 & - & 63,3 & 86,6 & * * \\
\hline 1980 & 12 & - & - & - & 4 & 5 & 8 & 8 & 4 & 1 & - & 70,0 & 83,3 & NS \\
\hline 1979 & 11 & - & - & 1 & 3 & 5 & 10 & 6 & 3 & 2 & - & 70,0 & 83,3 & ** \\
\hline 1978 & 10 & - & - & 2 & 2 & 5 & 9 & 7 & 4 & 1 & - & 70,0 & 83,3 & ** \\
\hline 1977 & 9 & 1 & 2 & - & - & 3 & - & 5 & 6 & 6 & 7 & 26,6 & 33,3 & NS \\
\hline 1976 & 8 & 2 & - & - & 1 & 3 & 4 & 3 & 5 & 5 & 7 & 33,3 & 40,0 & NS \\
\hline 1975 & 7 & 4 & - & - & 1 & - & 4 & 7 & 3 & 5 & 6 & 36,6 & 36,6 & IS \\
\hline 1974 & 6 & - & 2 & - & 3 & 2 & 3 & 2 & 6 & 5 & 7 & 23,3 & 30,0 & NS \\
\hline 1 & & & & & & & & & & & & & & \\
\hline 1973 & 5 & - & - & - & 2 & 3 & 4 & 2 & 7 & 6 & 6 & 30,0 & 26,6 & \\
\hline 1972 & 4 & - & - & 3 & 5 & - & 3 & 3 & 3 & 7 & 6 & 20,0 & 26,6 & \\
\hline 1971 & 3 & - & 2 & 1 & - & 4 & 4 & 6 & 5 & 4 & 4 & 46,6 & 30,0 & \\
\hline 1970 & 2 & - & - & 4 & 2 & 4 & 4 & 5 & 2 & 4 & 5 & 43,3 & 36,6 & S \\
\hline 1969 & 1 & - & - & 4 & 3 & 2 & 3 & 5 & 5 & 5 & 3 & 33,3 & 20,0 & \\
\hline
\end{tabular}

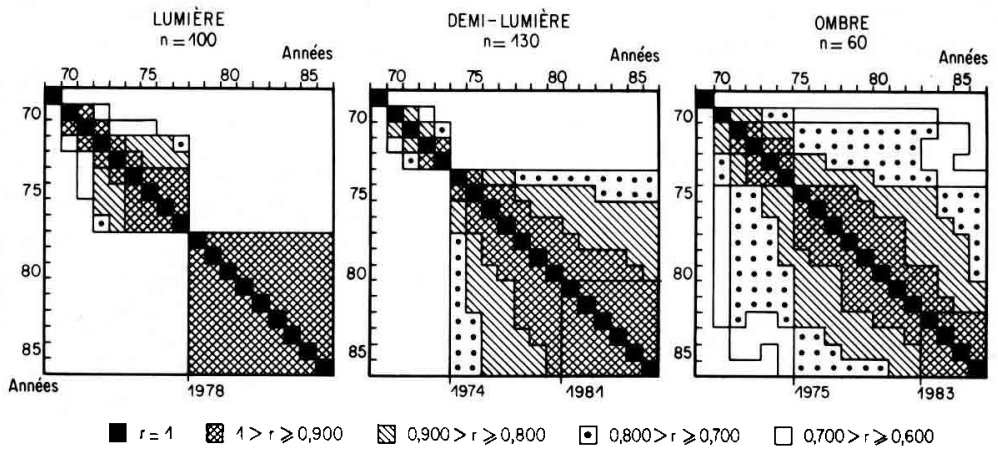

Fig 1. Évolution des classements annuels sous chaque éclairement pendant 18 ans. Coefficients de corrélations linéaires entre les années prises 2 à 2 . Coefficients supérieurs à 0,600 , tous significatifs avec un indice de sécurité $>0,01$. 
Tableau III. Lumière, classements annuels des 30 plants de la strate inférieure selon 10 classes de hauteurs. I: phase d'installation, II: de différenciation, III: de stabilisation (Thí́baut et al, 1992b). Proportion des 30 plants de la strate inférieure observés dans les 3 dernières classes, correspondant à cette strate (\%). $\chi^{2}$ : comparaison entre la répartition observée et une répartition régulière des 30 plants, en opposant les effectifs dans les 3 dernières classes à celui des 7 premières.

\begin{tabular}{|c|c|c|c|c|c|c|c|c|c|c|c|c|c|}
\hline \multirow[t]{2}{*}{ Années } & \multirow[t]{2}{*}{ Ages } & \multirow[b]{2}{*}{1} & \multirow[b]{2}{*}{2} & \multicolumn{6}{|c|}{ Classes de tailles décroissantes } & \multirow[b]{2}{*}{9} & \multirow[b]{2}{*}{10} & \multirow[t]{2}{*}{ (\%) } & \multirow[t]{2}{*}{$\left(x^{2}\right)$} \\
\hline & & & & 3 & 4 & 5 & 6 & 7 & 8 & & & & \\
\hline \multicolumn{14}{|l|}{ III } \\
\hline 1986 & 18 & - & - & - & - & - & - & - & 10 & 10 & 10 & 100,0 & *** \\
\hline 1985 & 17 & - & - & - & - & - & - & 1 & 9 & 10 & 10 & 96,6 & *** \\
\hline 1984 & 16 & - & - & - & - & - & - & 2 & 8 & 10 & 10 & 93,3 & $* * *$ \\
\hline 1983 & 15 & - & - & - & - & - & - & 2 & 8 & 10 & 10 & 93,3 & "** \\
\hline \multicolumn{14}{|l|}{ II } \\
\hline 1982 & 14 & - & - & - & - & - & - & 2 & 8 & 10 & 10 & 93,3 & \#** \\
\hline 1981 & 13 & - & - & - & - & - & 1 & 3 & 7 & 9 & 10 & 86,6 & $\star \star \star \star$ \\
\hline 1980 & 12 & - & - & - & - & 1 & 2 & 2 & 6 & 9 & 10 & 83,3 & *** \\
\hline 1979 & 11 & - & - & - & - & 1 & - & 4 & 7 & 8 & 10 & 83,3 & "**** \\
\hline 1978 & 10 & - & - & - & - & 1 & 1 & 3 & 6 & 9 & 10 & 83,3 & *** \\
\hline 1977 & 9 & 1 & 2 & 2 & 3 & 3 & 6 & 3 & 3 & 4 & 3 & 33,3 & NS \\
\hline 1976 & 8 & - & - & 3 & 3 & 4 & 4 & 4 & 4 & 5 & 3 & 40,0 & NS \\
\hline 1975 & 7 & - & 1 & 3 & 3 & 7 & 4 & 1 & 3 & 4 & 4 & 36,6 & NS \\
\hline 1974 & 6 & - & 2 & 2 & 4 & 5 & 4 & 4 & 3 & 4 & 2 & 30,0 & NS \\
\hline \multicolumn{14}{|l|}{1} \\
\hline 1973 & 5 & - & 3 & 5 & 4 & 4 & 3 & 3 & 1 & 3 & 4 & 26,6 & NS \\
\hline 1972 & 4 & - & 4 & 3 & 2 & 4 & 5 & 4 & 2 & 2 & 4 & 26,6 & NS \\
\hline 1971 & 3 & 3 & 3 & 2 & 6 & 3 & 2 & 2 & 2 & 3 & 4 & 30,0 & NS \\
\hline 1970 & 2 & 3 & 3 & 3 & 3 & 2 & 3 & 2 & 5 & 2 & 4 & 36,6 & NS \\
\hline 1969 & 1 & 2 & 5 & 3 & 2 & 5 & 3 & 4 & 1 & 2 & 3 & 20,0 & NS \\
\hline
\end{tabular}

dement dans l'intervalle d'une année à la lumière. II est plus tardif et plus progressif en demi-lumière où il se réalise au bout de 7 années. Enfin, ce classement se dessine encore plus tard et de manière plus progressive à l'ombre au bout de 8 années.

\section{Classements annuels selon la classe des plants en 1986}

La diversité des comportements individuels est très grande. Cependant des analyses en composantes principales révèlent des tendances selon les individus et selon les années. Sous chaque éclairement, le comportement des plants a été décrit selon leurs classements annuels, pendant 18 ans.

À la lumière et en demi-lumière, l'axe (I) représente surtout la variation interclasse et l'axe (II) la variation intra-classe (fig 2). Cette dernière paraît assez importante, en sorte que les classes se superposent partiellement. Cependant leur agencement selon l'axe (I) traduit bien une évolution progressive de la plus grande classe a la plus petite. A la lumière, les classes appartenant à la strate supérieure et inférieure, sont disjointes. En demi-lumière, les 2 


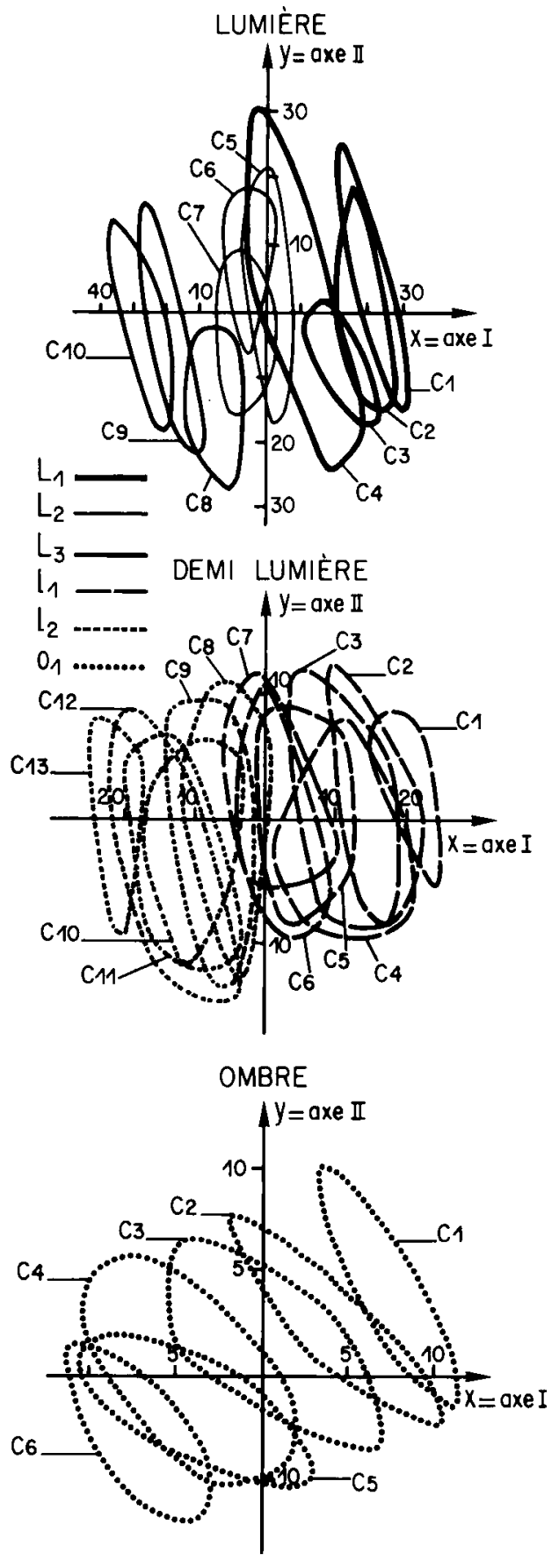

strates se recouvrent partiellement. $\AA$ l'ombre, la composante intraclasse est toujours importante; les classes extrêmes ne se recouvrent pas et elles sont toutes ordonnées selon la bissectrice de l'angle formé par les 2 axes. Ainsi, les tendances sont les mêmes quels que soient l'éclairement et la structure de la régénération, mono- ou pluristrate. Le comportement des plants dépend surtout de leur classement final.

À la lumière, les années se regroupent en 2 périodes distinctes avant et après 1978 (fig 3). En demi-lumière, la même tendance apparaît avant et après 1974. Alors qu'à l'ombre, les années se succèdent dans un ordre chronologique sans marquer de regroupement net. Ces résultats confirment ceux qui sont décrits dans la figure 1. Ces différences peuvent être dues à la stratification et/ou à l'éclairement.

\section{Recrutements des plants dans leur strate-86 et dans leur classe-86}

Le classement définitif ou le recrutement des plants dans leur strate et dans leur classe ont été décrits sous chaque éclairement (figs $4,5,6$ ). Comme on pouvait s'y attendre, les plants sont recrutés plus rapidement dans leur strate- 86 qui comprend

Fig 2. Classements annuels selon les individus sous chaque éclairement, analyse en composantes principales. Métrique des individus : à la lumière $N=100$, en demi-lumière $N=120$ et à l'ombre $N=60$, à raison de 10 individus par classe de hauteur sous chaque éclairement. Tous les individus ont le même poids. Métrique des variables : 18 variables correspondant aux années. Interties absorbées à la lumière, axe I: $60,85 \%$ et axe II : $26,10 \%$; en demi-lumière, axe I : $70,43 \%$ et axe II : $15,90 \%$; à l'ombre, axe I : $78,10 \%$ et axe $\|: 9,07 \%$. De $C_{1}$ à $C x$, classes86 de hauteurs des plus grands aux plus petits. 

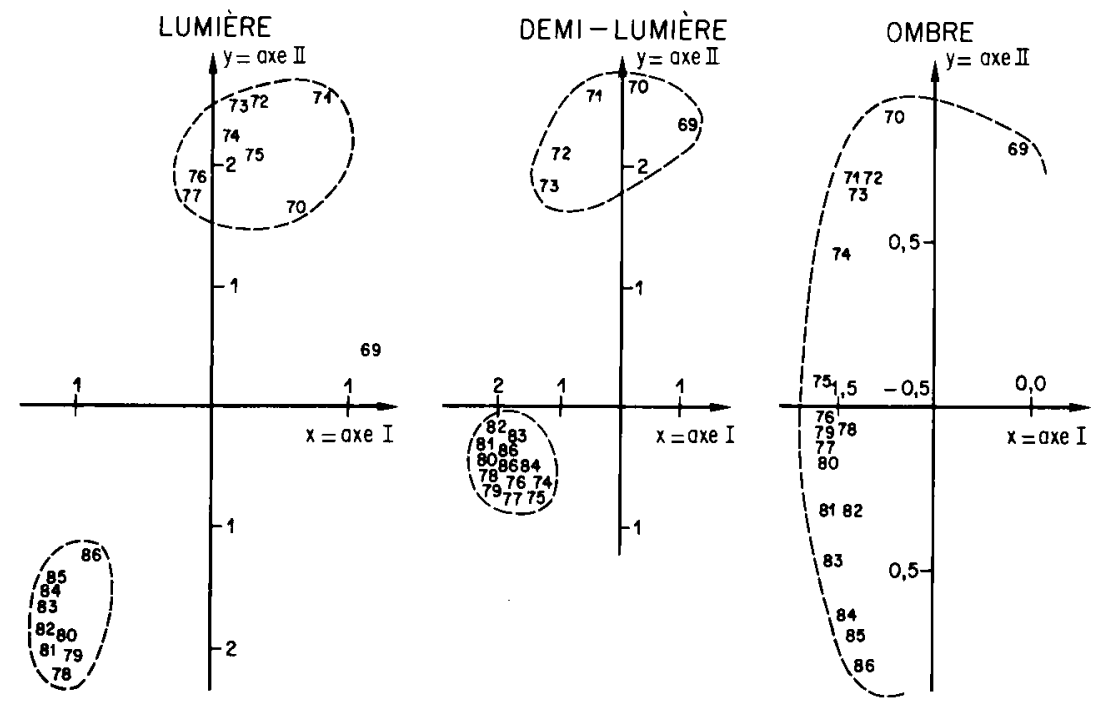

Fig 3. Classements annuels selon les années sous chaque éclairement, analyse en composantes principales. Métrique des individus : à la lumière $N=100$, en demi-lumière $N=120$ et à l'ombre $N=$ 60 , à raison de 10 individus par classe de hauteur sous chaque éclairement. Tous les individus ont le même poids. Métrique des variables : 18 variables correspondant aux années. Inerties absorbées à la lumière, axe I : $60,85 \%$ et axe II : $26,10 \%$; en demi-lumière, axe I: $70,43 \%$ et axe II : $15,90 \%$; à l'ombre, axe I : $78,10 \%$ et axe II : $9,07 \%$.

plusieurs classes que dans leur classe-86. En effet, lorsqu'un sujet est recruté dans sa strate, il peut encore présenter des reclassements à l'intérieur de celle-ci.

Sous les 3 éclairements, les recrutements sont plus précoces dans les classes-86 extrêmes, celles des plus grands et des plus petits, alors qu'ils sont plus tardifs dans les classes intermédiaires. Donc, les recrutements sont bien liés à la vigueur des plants en 1986. Cette règle est identique que la structure soit mono- ou pluristrate.

À la lumière, figure 4 , les recrutements dans les classes- 86 débutent souvent entre les années 1977-1978, alors que les recrutements dans la strate sont pratiquement achevés à la même date dans la strate supérieure, sauf pour les plants de la classe $\mathrm{C} 4$ et dans la strate inférieure, sauf pour les plants de la classe C8. En demi-lumière (fig 5) et à l'ombre (fig 6), les recrutements sont plus progressifs, sans que les années 1977-1978 aient un impact particulier. Les recrutements sont accélérés par la lumière et la stratification n'a aucune influence sur eux.

\section{Mouvements des plants selon leur classe en 1986}

Le nombre des reclassements ne varie guère selon la vigueur-86 des plants à la lumière (tableau IV). II diminue légèrement dans les classes extrêmes en demi- 

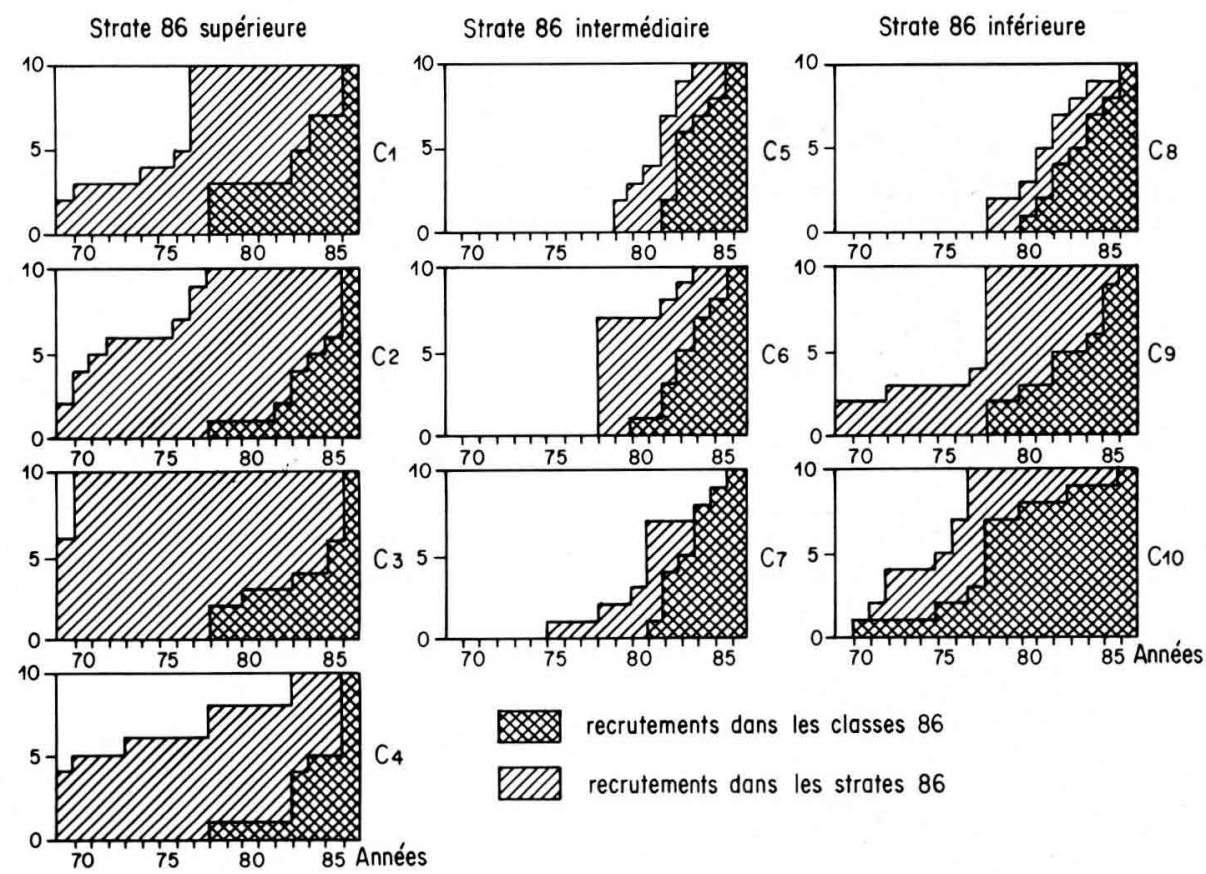

recrutements dans les classes 86

DIIA recrutements dans les strates 86

Fig 4. Recrutements des plants dans leur strate- 86 et dans leur classe- 86 à la lumière. $\mathrm{De} \mathrm{C}_{1}$ à $\mathrm{C}_{10}$, classes-86 de hauteur des plus grands aux plus petits.

lumière. Par contre, il est nettement plus faible chez les plants les plus vigoureux à l'ombre (tableau VI).

L'amplitude moyenne de ces mouvements ne varie pas beaucoup selon la classe-86. Par contre, leur nature change. À la lumière et en demi-lumière, les promotions sont significativement plus nombreuses que les déclassements dans les classes supérieures et, au contraire, les déclassements l'emportent dans les classes inférieures. Donc, la nature des mouvements est bien liée à la vigueur des plants en 1986 sous ces 2 éclairements. Aucune tendance n'apparaît à l'ombre. Là encore, la stratification n'a pas d'influence sur le nombre et l'ampleur des mouvements observés.

\section{Mouvements des plants selon les années}

Le nombre moyen de reclassements par individu entre 2 années consécutives et le nombre moyen de classes concernées par mouvement ont été calculés selon l'éclairement et selon la strate (fig 7).

Les reclassements n'évoluent pas de la même manière au cours des années sous les 3 éclairements. À la lumière et dans les 3 strates, la majorité des reclassements se sont produits en très peu d'années, pendant 2 périodes distinctes : au cours des 3 premières années (1969 à 1972) puis entre 1977 et 1978. Sous les 2 autres éclairements, la plupart des reclassements 


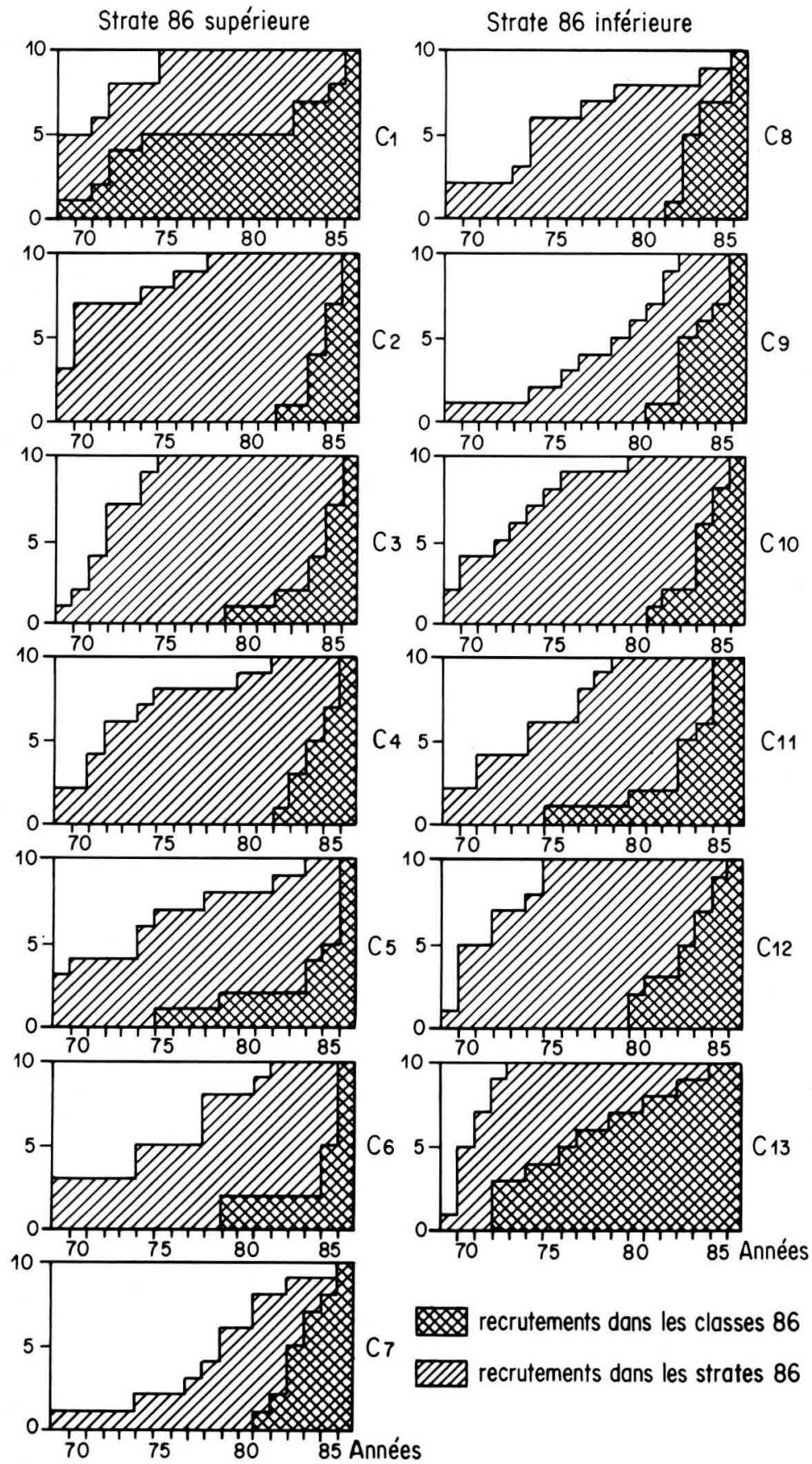

Fig 5. Recrutements des plants dans leur strate-86 et dans leur classe-86 en demi-lumière. $D_{\theta} C_{1}$ à $\mathrm{C}_{13}$, classes-86 de hauteur des plus grands aux plus petits. 


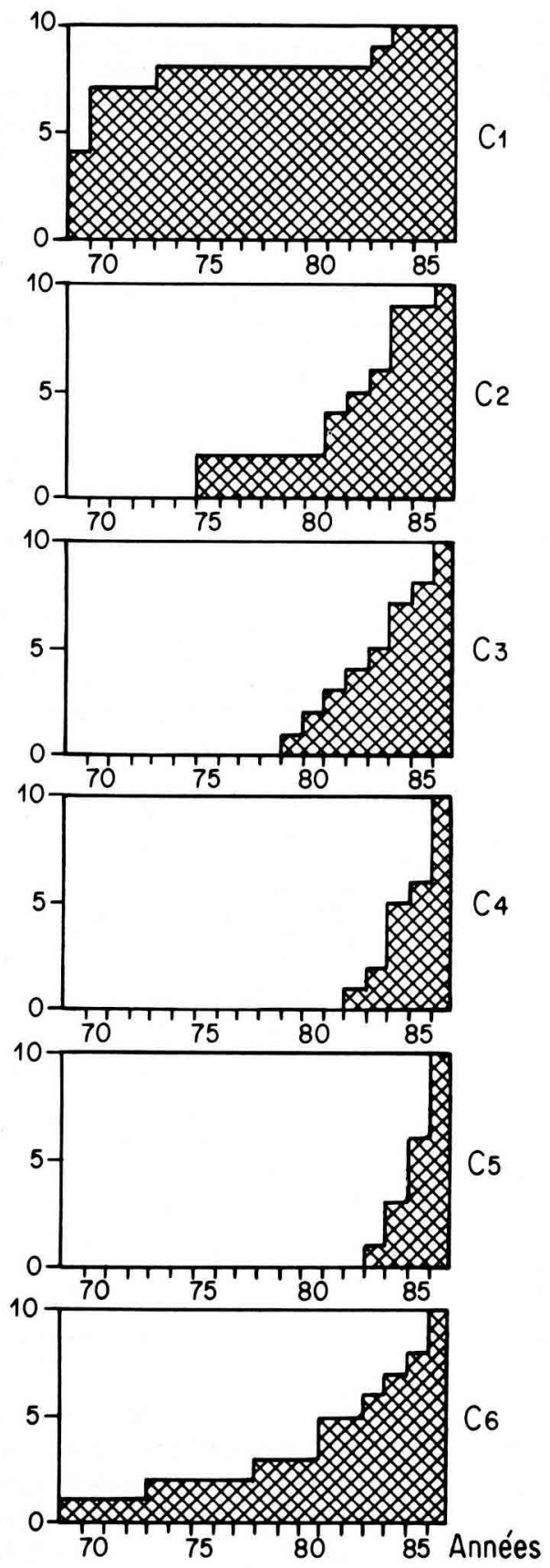

recrutements dans les classes 86 se réalisent uniquement au début mais ils sont plus étalés en demi-lumière qu'à l'ombre. La stratification semble encore sans influence sur les mouvements.

\section{DISCUSSION}

\section{Structure pluristrate et hiérarchie sociale}

La stabilité des structures pluristrates est souvent affirmée mais rarement démontrée (Delvaux, 1964; Richter, 1971). Dans une plantation de douglas, ce dernier auteur analyse les reclassements des individus entre 3 et 7 ans, pendant 4 années consécutives. II constate que les hauteurs initiales et finales sont fortement corrélées et que $35-72 \%$ des sujets se maintiennent dans la même classe de hauteur relative. L'auteur conclut à la stabilité des plantations mais ne se prononce pas sur l'importance des reclassements avant 3 ans et après 7 ans. Au Lingas, nous avons analysé les reclassements dans une régénération naturelle de hêtre sur une période plus longue. Les plants appartenant à une classe de hauteur en 1986 sont recrutés parmi toutes les autres classes au cours des 5 premières années pendant la phase d'installation (tableaux I-III). Les nombres de promotions et de déclassements s'équilibrant, aucun tri ne se produit pendant cette période (tableau IV). Puis des regroupements se dessinent entre 5 et 10 ans, au début de la phase de différenciation. Les plants sont triés plus ou moins rapidement selon l'éclairement et selon leur vigueur-86 (figs 4-6). Enfin, après, les

Fig 6. Recrutements des plants dans leur strate86 et dans leur classe-86 a l'ombre. $\mathrm{De} \mathrm{C}_{1}$ a $\mathrm{C}_{6}$, classes-86 de hauteur des plus grands aux plus petits. 
Tableau IV. Reclassements des plants selon l'éclairement et selon leur classe-86. $\chi^{2}$ dans une classe-86, comparaison des gains et des pertes observés à ceux attendus si répartition équitable des classes impliquees entre les gains et les pertes. $|x|$ : importance moyenne par reclassement.

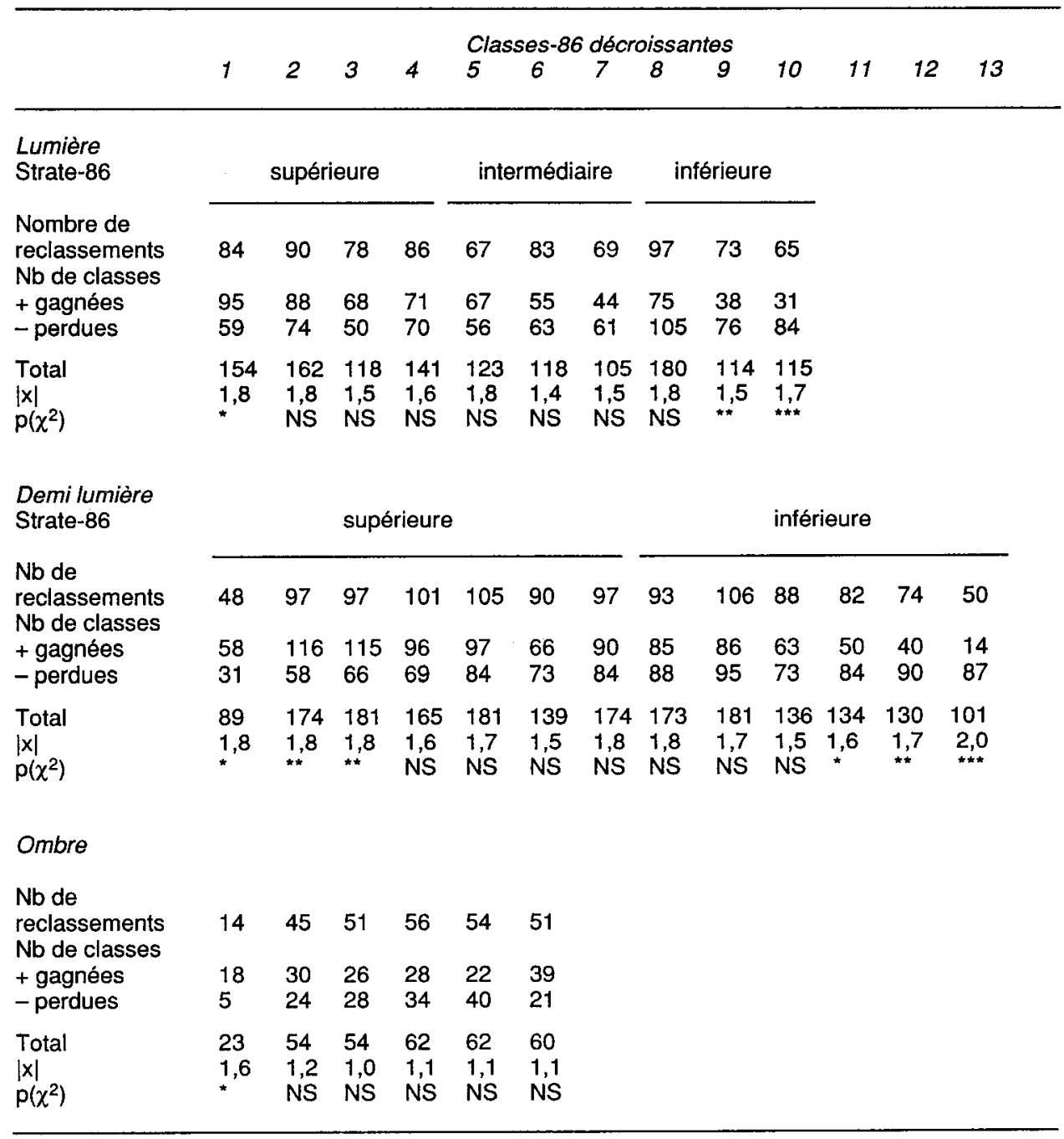

plants se maintiennent dans leur classe définitive. L'analyse des reclassements, en nombre et en ampleur, révèle leur importance au cours des premières années mais aussi leur brièveté dans le temps, notamment à la lumière (fig 7). Et, indépen- damment de la structure horizontale de la régénération, les recrutements sont toujours plus rapides pour les classes de hauteurs extrêmes alors qu'ils sont lents pour les classes intermédiaires (figs 4-6). Finalement, la stratification ne semble pas 
Reclassements des plants de hêtre
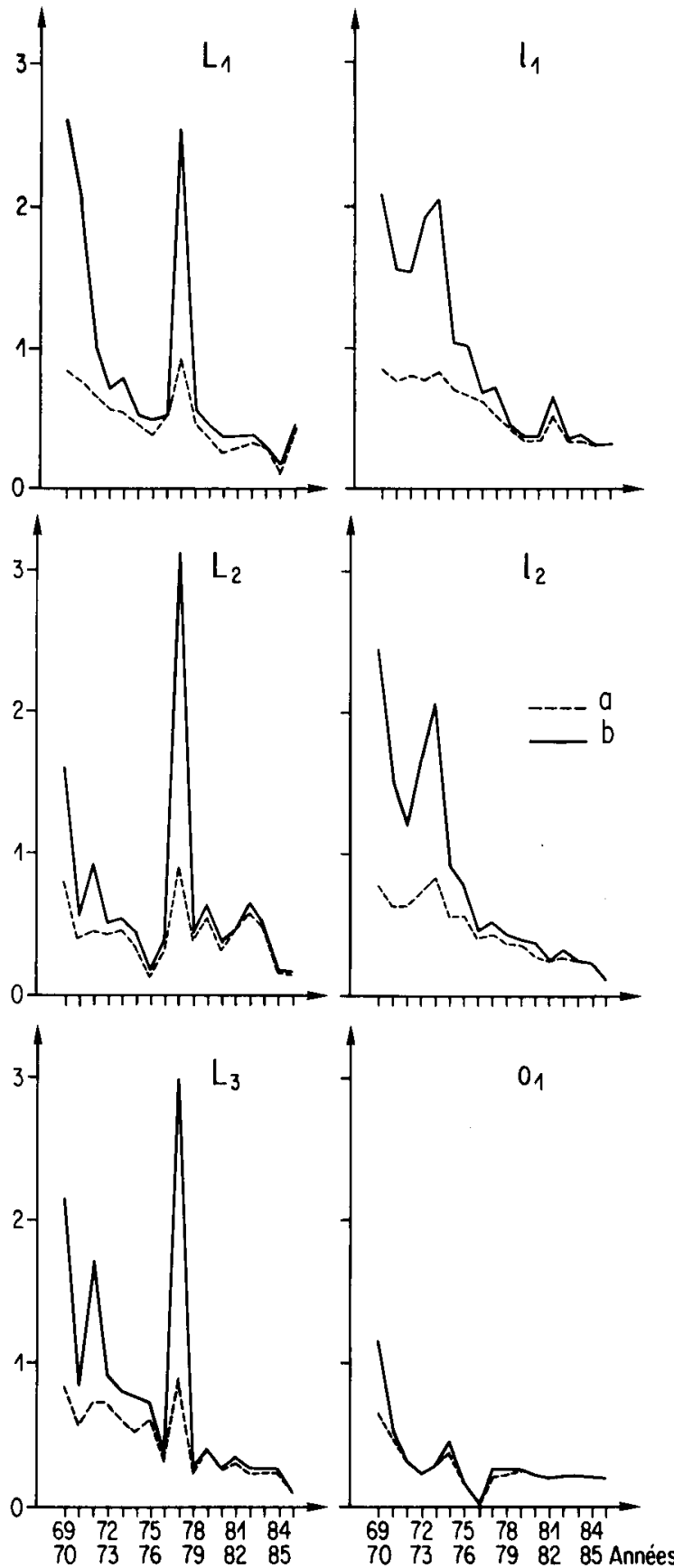

Fig 7. Mouvements des plants selon l'éclairement et la strate au cours des 18 ans. a. nombre moyen de reclassements par individu entre 2 années consécutives, b. nombre moyen de classes par reclassement entre 2 années consécutives. 
avoir une grande influence sur ces mouvements dont le nombre, l'ampleur et la brièveté dans le temps dépendent plutôt de l'éclairement et de la vigueur des plants à 18 ans. Donc, si la stratification a un effet sur la vigueur et la forme des plants à 18 ans et sur leur développement au cours des 18 premières années (Thiébaut et al, 1992), elle semble sans influence sur leurs reclassements qui n'offrent donc aucun argument supplémentaire pour pouvoir assimiler la stratification à une forme de hiérarchie sociale.

\section{Fonctionnement d'une régénératon équienne de hêtre}

Comme nous venons de le voir au Lingas et surtout à la lumière, la destinée d'une régénération et du futur peuplement se joue en quelques années après l'installation du semis. On distingue rapidement une population principale et une population subordonnée (Badoux, 1939; Delvaux, 1964, 1975; Falcone, 1985; Lanier, 1986; Falcone et al, 1986; Gauthier, 1987).

Cependant, ce schéma général doit être modulé. En effet, un premier classement peut-être remis en cause, comme cela a été nettement le cas à la lumière, et les reclassements doivent se poursuivre au-delà de 18 ans. En effet, tous les plants de la strate supérieure ne parviendront pas au stade adulte et des déclassements doivent se produire par la suite. Certains auteurs signalent des reclassements à des âges plus avancés : entre 25 et 40 ans (Schädelin, 1937), entre 10-20 ans et 50 ans (Pardé et Venet, 1981), entre 37 et 40 ans (Bouchon et al, 1989a,b) vers 32 ans (Ung, 1989 sur Fagus grandifolia). Ces déclassements rendent difficile le repérage des sujets les mieux adaptés à long terme alors qu'il est plus facile d'indiquer les sujets incompétents (Schütz, 1981).
Ainsi, l'évolution des peuplements se produirait en 4 périodes, nos observations portant uniquement sur les 3 premières (Thiébaut et al, 1992):

- une phase d'installation sans disparités morphologiques d'une strate à l'autre, caractérisée par de nombreux reclassements entre toutes les classes, avec autant de promotions que de déclassements, jusqu'à 5-6 ans au Lingas;

- une phase de différenciation caractérisée par l'apparition de disparités morphologiques entre les strates et par des reclassements orientés, entre 6 et 15 ans;

- une phase de stabilisation caractérisée par une diminution de la croissance annuelle, le maintien des disparités morphologiques entre les strates et la fin des reclassements, entre 15 et 18 ans;

- enfin, une phase relativement longue si l'on en juge par les résultats des auteurs précités, au cours de laquelle s'effectueraient des déclassements essentiellement dans la strate supérieure.

Une compétition excessive freine la croissance des plants, y compris celle des plus grands. Les dépressages sont donc nécessaires pour libérer les individus et favoriser le développement des houppiers comme le pensent de nombreux forestiers (Schädelin, 1937; Delvaux, 1964; Leibundgut, 1971; Keller et al, 1976; Polge, 1981 et 1983; Schütz, 1981; Lanier, 1981 a,b; Ferrand, 1982; Bouchon et al, 1989a,b).

\section{REMERCIEMENTS}

Travail réalisé avec l'aide de I'INRA, contrat "Amélioration, sylviculture et qualité du bois des feuillus précieux», $n^{\circ} 1233 A$. Nous exprimons nos remerciements à Monsieur $E$ Teissier-duCros et aux 2 lecteurs des Annales des Sciences Forestières pour leurs remarques ayant contribué à améliorer la rédaction de ce texte. 


\section{RÉFÉRENCES}

Badoux E (1939) De l'influence de divers modes et degrés d'éclaircies dans les hêtraies pures. Mitt Schweiz Anst Forst/ Versuchswes 21, 59-145

Bouchon J, Dhote JF, Lanier L (1989a) Note sur la réaction individuelle du hêtre à différentes intensités d'éclaircie et à différents âges. Rev For Fr 41, 39-50

Bouchon J, Dhote JF, Lanier L (1989b) Réaction individuelle de hêtres (Fagus sylvatica $\mathrm{L}$ ) d'âges divers à diverses intensités d'éclaircie. Ann Sci For 46, 251-259

Delvaux J (1964) Contribution à l'étude de l'édu cation des peuplements. I. Acquisition de la position dominante dans les jeunes plantations équiennes d'épicéa. Stn Rech Eaux For, Trav Ser B, $n^{\circ} 29,38 \mathrm{p}$

Delvaux J (1975) Contribution à l'étude de l'éducation des peuplements. XIV. Acquisiton du rang social dans les jeunes plantations d'épicéa. Stn Rech Eaux For, Trav Ser B, 39, $32 \mathrm{p}$

Falconne $P$ (1985) Structure, croissance et aspects qualitatifs des plantations de hêtre (Fagus silvatica L). Mémoire $3^{\theta}$ année, ENITEF, Station de sylviculture et de production INRA, CNRF (doc 85/01), Nancy, $90 p$

Falcone $P$, Keller R, Le Tacon F, Oswald $H$ (1986) Facteurs influençant la forme des feuillus en plantation. Rev For Fr 38, 315-323

Ferrand JC (1982) Étude des contraintes de croissance. 2. Variabilité en forêt des contraintes de croissance du hêtre (Fagus sylvatica L). Ann Sci For 39, 187-218

Gauthier C (1987) Amélioration des formes et comportements des plantations de hêtre (Fagus sylvatica L) en haute Normandie. Des solutions possibles. Mémoire $3^{\theta}$ année, ENITEF, ENGREF, CIHEAM, FFSRC, $45 \mathrm{p}$

Keller R, Le Tacon F, Timbal J (1976) La densité du bois de hêtre dans le Nord-Est de la France. Influence des caractéristiques du milieu et du type de sylviculture. Ann Sci For 33, 1-17

Lanier $L$ (1981a) Les dégagements et nettoiements en futaie feuillue. Rev For Fr 33, $\mathrm{n}^{\circ}$ $\mathrm{sp}, 19-40$
Lanier L (1981b) Conditions de germination des faines, de survie et de croissance des semis. Coupes de mise en lumière. In: Le Hêtre (Teissier-du-Cros E, Le Tacon F, Nepveu G, Pardé J, Perrin R, Timbal J, eds), INRA, Rech For Paris, 239-241

Lanier L (1986) Précis de sylviculture. Écol Nat Génie Rural Eaux For, Nancy, 468 p

Leibundgut $H$ (1971) Ergebnisse von Durchforstungsversuchen 1930-1965 im Sihlwald. Mitt Schweiz Anstalt Forstliche Versuchswesen 47, 259-389

Pardé J, Venet J (1981) Conduite des peuplements. In: Le hêtre (Teissier-du-Cros $\mathrm{E}, \mathrm{Le}$ Tacon F, Nepveu G, Pardé J, Perrin R, Timbal J, eds), INRA, Dép Rech For Paris, 272293

Polge H (1981) Influence des éclaircies sur les contraintes de croissance du Hêtre. Ann Sci For $38,407-423$

Polge $H$ (1983) Essai de ligniculture du Hêtre à partir de régénération naturelles denses. Bull Tech ONF 14, 11-17

Richter J (1971) Das Umsetzen von Douglasien im Kulturstadium. Allg Forst Jagdzg 142 jhrg, H3, 65-69

Schädelin W (1937) L'éclaircie. Traitement des forêts par la sélection qualitative. Attinger, Neuchâtel, $110 \mathrm{p}$

Schütz JP (1981) L'éclaircie sélective de Schädelin, évolution et pratique actuelle. Rev For Fr 33, $n^{\circ}$ sp 7-18

Schütz JP (1990) Sylviculture 1, principes d'éducation des forêts. Collection gérer l'environnement, Presses Polytech et Univ Romandes, Suisse, $243 p$

Thiébaut B, Comps B, Rucart M, Soroste S, Nitsame Okwo C (1992) Développement des plants de hêtre (Fagus sylvatica $L$ ) dans une régénération naturelle, équienne, âgée de 18 ans. Ann Sci For 49, 111-131

Ung $\mathrm{CH}$ (1989) Forme des tiges d'érable à sucre et de hêtre à grandes feuilles dans une jeunes futaie selon leur position sociale et leur âge. Ann Sci For 46, 261-271 